\title{
Prediction of a Photon Peak in Relativistic Heavy Ion Collisions
}

\author{
Jorge Casalderrey-Solana ${ }^{1}$ and David Mateos ${ }^{2}$ \\ ${ }^{1}$ Nuclear Science Division, MS 70R319, Lawrence Berkeley National Laboratory, Berkeley, California 94720, USA \\ ${ }^{2}$ Department of Physics, University of California, Santa Barbara, California 93106-9530, USA
}

(Received 16 July 2008; published 14 May 2009)

\begin{abstract}
We show that if a flavorless vector meson remains bound after deconfinement, and if its limiting velocity in the quark-gluon plasma is subluminal, then this meson produces a distinct peak in the spectrum of thermal photons emitted by the plasma. We also demonstrate that this effect is a universal property of all strongly coupled, large- $N_{c}$ plasmas with a gravity dual. For the $J / \psi$, the corresponding peak lies between 3 and $5 \mathrm{GeV}$ and could be observed in heavy-ion collisions at the LHC.
\end{abstract}

1. Introduction.-At $T_{c} \simeq 170 \mathrm{MeV}$, quantum chromodynamics $(\mathrm{QCD})$ undergoes a rapid crossover into a deconfined phase called the quark-gluon plasma (QGP). This state of matter is being intensively studied at the Relativistic Heavy Ion Collider (RHIC) and will be studied in the future at the Large Hadron Collider (LHC).

A remarkable conclusion from the RHIC experiments is that the QGP does not behave as a weakly coupled gas of quarks and gluons but rather as a strongly coupled fluid. This makes the study of the plasma a challenging task. Experimentally, it is difficult to find direct probes with which to determine the properties of the plasma, since any strongly interacting probe will couple to the medium in a complicated manner; for this reason, thermal photons emitted by the plasma are particularly interesting. Predicting the properties of the QGP from first principles is also challenging, since the strong coupling renders perturbative methods inapplicable in general. Lattice QCD is also of limited utility, since it is not well suited for studying real-time phenomena such as photon production. Thus one must either make predictions based on very general expectations or resort to some other nonperturbative method such as the gauge-gravity duality.

In this Letter, we will show that, under two plausible assumptions about the properties of heavy mesons in the QGP, a distinct peak in the spectrum of thermal photons is predicted. Moreover, we will demonstrate that this is a universal property of all strongly coupled, large- $N_{c}$ theories with a gravity dual. Finally, we discuss under what conditions this effect could be observed at the LHC.

2. Peaks in the photon spectrum.-Sufficiently heavy mesons (e.g., $J / \psi, Y$, etc.) may be expected to exhibit two generic properties in the QGP. First, they may remain bound up to a dissociation temperature $T_{\text {diss }}>T_{c}$. Second, their limiting velocity in the plasma may be subluminal.

The original argument [1] for the first expectation is simply that the heavier the meson, the smaller its size. It is thus plausible to expect a meson to remain bound until the screening length in the plasma becomes comparable to the meson size, and for sufficiently heavy mesons this happens at $T_{\text {diss }}>T_{c}$. This conclusion is supported by calculations of both the static quark-antiquark potential [2] and of Minkowski-space spectral functions in latticeregularized QCD [3].

The second expectation goes back to Ref. [4], which considered a weakly coupled plasma. More generally, one may note [5] that a meson moving in the plasma with velocity $v$ experiences a boosted, higher energy density and hence also a higher effective temperature $T_{\text {eff }}(v)=$ $\left(1-v^{2}\right)^{-1 / 4} T$. Thus one may expect a subluminal limiting velocity for the meson [6] determined by the condition $T_{\text {eff }}\left(v_{\text {lim }}\right) \sim T_{\text {diss }}[5]$.

Although the two assumptions above are reasonable, by no means have they been rigorously established in QCD. Our purpose is not to discuss their plausibility in detail but to exhibit an immediate consequence.

Consider the in-medium meson dispersion relation (DR) $\omega(k)$, where $\omega$ and $k$ are the energy and the spatial threemomentum of the meson, respectively. $M=\omega(0)$ is the rest mass. As $k \rightarrow \infty$, the assumption of a subluminal limiting velocity implies $\omega(k) \sim v_{\lim } k$, with $v_{\lim }<1$. Figure 1 shows the DR for a pseudoscalar meson in the strongly coupled, large- $N_{c}$, four-dimensional, $\mathcal{N}=4$ super Yang-Mills plasma, calculated using its gravity

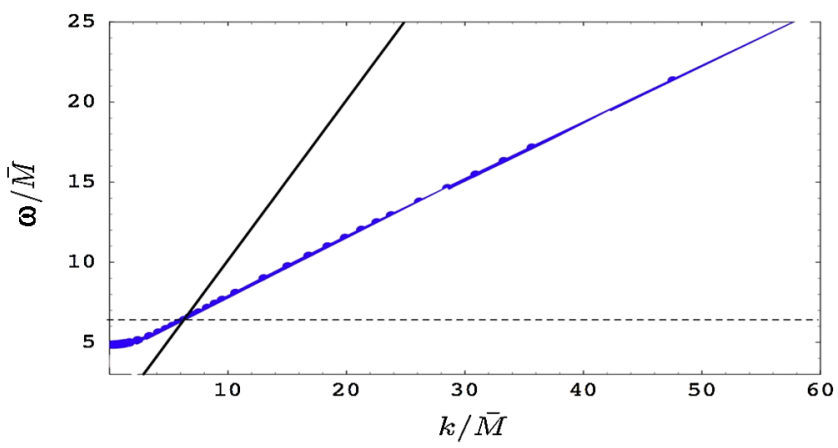

FIG. 1 (color online). Dispersion relation (blue curve) for a heavy meson [7]. The black straight line corresponds to $\omega=k$. $\bar{M}$ is a reference scale. 


\section{Meson $\longrightarrow \gamma$}

FIG. 2. Decay of a vector meson into an on-shell photon.

dual [7]; the DR for vector mesons is expected to exhibit the same features. As noted in Ref. [8], continuity now implies that the DR curve must cross the light cone, defined by $\omega=k$, at some energy $\omega=\omega_{\text {peak }}$. At this point the meson four-momentum is null, and so the meson possesses the same quantum numbers as a photon [9]. Such a meson can then decay into an on-shell photon [10]; see Fig. 2. This process contributes a resonance peak, at an energy $\omega_{\text {peak }}$, to the in-medium spectral function of two electromagnetic currents $\chi_{\mu \nu}(\omega, k) \sim\left\langle J_{\mu}(\omega, k) J_{\nu}(-\omega,-k)\right\rangle$ evaluated at null-momentum $\omega=k$. This in turn produces a peak in the spectrum of thermal photons $d N_{\gamma} / d \omega \sim$ $e^{-\omega / T} \chi^{\mu}{ }_{\mu}(\omega, T)$, whose width is the width of the meson in the plasma. Figure 3 illustrates this effect for the $\mathcal{N}=4$ plasma. The results are valid at strong coupling and large $N_{c}$, since they were obtained by means of the gravity dual [8]. The spectral function for the massless quark is structureless, whereas that for the heavy quark exhibits a resonance peak.

3. A universal property of plasmas with a gravity dual.The gravity dual of QCD is presently unknown. It is therefore important to focus on properties of the gauge-gravity duality that apply to as broad a class of plasmas as possible, since these may also apply to QCD. Here we will show that the two assumptions about heavy mesons in a QGP are true in all strongly coupled, large- $N_{c}$ plasmas with a gravity dual, because they follow from two universal properties of the duality: (i) The deconfined phase is described by a black hole $(\mathrm{BH})$ geometry [11], and (ii) a finite number of flavors $N_{f}$ is described by $N_{f}$ D-brane probes in this geometry [12].

In the presence of the $\mathrm{BH}$, there are two possible phases separated by a universal first-order phase transition $[13,14]$. In one phase, the branes lie completely outside the horizon in a "Minkowski embedding" (see Fig. 4). In

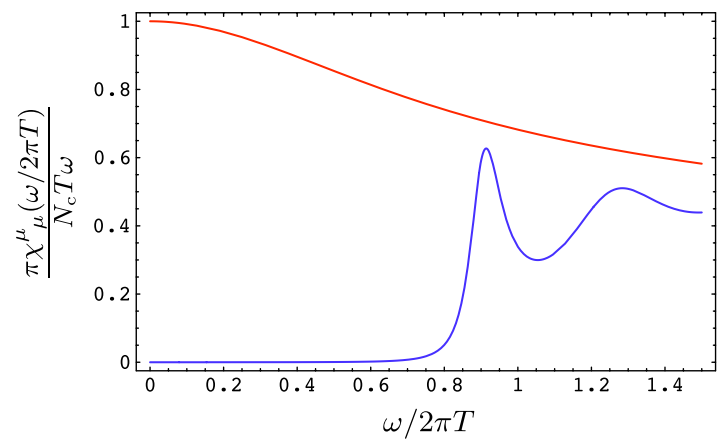

FIG. 3 (color online). Spectral functions for the $\mathcal{N}=4$ plasma coupled to a massless quark (top, red curve) and a heavy quark (bottom, blue curve). the other phase, they fall through the horizon in a "BH embedding." In the gauge theory, the phase transition corresponds to the dissociation of heavy mesons $[13,18]$. In the Minkowski phase, stable mesons exist, and the meson mass increases with the branes-BH separation [19]. In the $\mathrm{BH}$ phase, no meson bound states exist. Recalling that the $\mathrm{BH}$ radius is proportional to the plasma temperature, we see that if a meson is sufficiently heavy compared to the temperature, then this meson remains bound and is described by a Minkowski brane.

The existence of a subluminal limiting velocity for mesons is now obvious [7]. Indeed, the wave function of a meson is supported on the D-branes. The larger its energy, the more the meson is attracted by the black hole and the more its wave function is concentrated at the tip of the branes (see Fig. 4). In the limit $k \rightarrow \infty$, the velocity of this meson approaches the local speed of light at the tip of the branes. Because of the redshift caused by the $\mathrm{BH}$, this limiting velocity is lower than the speed of light at infinity. In the gauge theory, this translates into the statement that $v_{\text {lim }}$ is lower than the speed of light in the vacuum [7]. This effect is clearly seen in Fig. 1.

4. Heavy-ion collisions.-Our analysis so far applies to an infinitely extended plasma at constant temperature. A crucial question is whether a photon peak could be observed in a heavy-ion collision experiment. Natural heavy vector mesons to consider are the $J / \psi$ and the $Y$, since these are expected to survive deconfinement. We wish to compare the number of photons coming from these mesons to that coming from other sources. Accurately calculating the meson contribution would require a precise theoretical understanding of the dynamics of these mesons in the QGP, which at present is not available. Our goal will therefore be to estimate the order of magnitude of this effect with a simple model.

Following Ref. [21], we model the fireball as an expanding cylinder with volume $V(t)=\pi\left(z_{0}+v_{z} t\right) \times$ $\left(r_{0}+a_{\perp} t^{2} / 2\right)^{2}$; the ratio $t_{\text {therm }} \equiv z_{0} / v_{z}$ is a measure of the thermalization time. The temperature evolution is $T(t)=T(0)[V(0) / V(t)]^{1 / 3}$. At $t=t_{\text {hadro }}$, the temperature reaches $T_{c}$ and the system hadronizes.

Let us first consider the $J / \psi$ contribution. In the spirit of statistical recombination [22,23], we assume that the $c \bar{c}$ pairs produced in the initial partonic collisions become
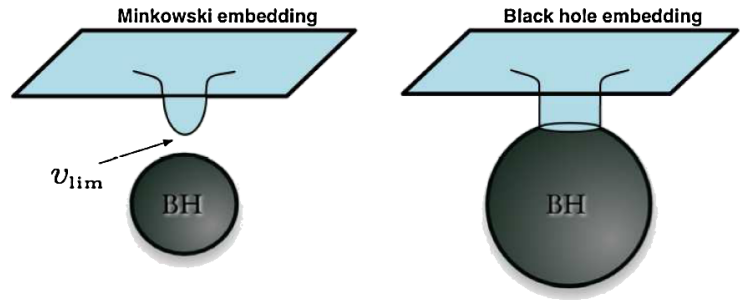

FIG. 4 (color online). Possible D-brane embeddings in a $\mathrm{BH}$ background. 
kinetically but not chemically equilibrated in the QGP [24]; in particular, their total number $N_{c \bar{c}}$ stays constant. To implement this condition, we introduce a fugacity factor $[22,23]$

$$
g_{c}(T)=\frac{N_{c \bar{c}}}{2 \times 3 \times V(T)\left(\frac{M_{c} T}{2 \pi}\right)^{3 / 2} e^{-M_{c} / T},}
$$

where $M_{c}$ is the in-medium charm-quark mass. At $t=t_{\text {diss }}$, the temperature reaches the dissociation temperature of the $J / \psi$ in the medium, $T_{\text {diss }}$. At this point a fraction of the $c \bar{c}$ pairs coalesce and recombine forming $J / \psi$ mesons [25]. Their contribution to the total number of photons with frequency $\omega$ emitted by the plasma is

$$
S(\omega) \propto \int_{t_{\text {diss }}}^{t_{\text {hadro }}} d t V(t) g_{c}[T(t)]^{2} e^{-\omega / T(t)} \chi_{J / \psi}(\omega, T(t)),
$$

where the " $S$ " stands for "signal." The overall normalization is not important since it is the same as that for the background of thermal photons emitted by the light quarks, which is given by

$$
B(\omega) \propto \int_{0}^{t_{\text {hadro }}} d t V(t) e^{-\omega / T(t)} .
$$

We have omitted $\chi_{\text {light }}(\omega, T)$ since, guided by the results for plasmas with a gravity dual, we assume that it is structureless.

We would like to compare the two contributions above. Motivated by Fig. 3, we expect the magnitude of $\chi_{J / \psi}$ around $\omega_{\text {peak }}$ to be comparable to that of $\chi_{\text {light }}$. We thus model the spectral function for the $J / \psi$ by a unit-area Gaussian distribution of width $\Gamma$ centered at $\omega_{\text {peak }}(T(t))$. For the width, we choose $\Gamma=100 \mathrm{MeV}$, of the order of the temperature and a thousand times larger than the vacuum width. The temperature dependence of $\omega_{\text {peak }}$ arises from that of the meson DR. On general grounds, we expect this to take the form $\omega(k)=\sqrt{M^{2} v_{\lim }^{4}+k^{2} v_{\lim }^{2}}+M\left(1-v_{\mathrm{lim}}^{2}\right)$. The first term is just the vacuum DR with the speed of light replaced by $v_{\text {lim }}$. The second term ensures that $M$ is the rest mass of the meson. The meson DR in Fig. 1 is very well approximated by this ansatz. We neglect medium-induced changes in the $J / \psi$ mass and set $M=3 \mathrm{GeV}$. The above ansatz yields $\omega_{\text {peak }}=M\left(1+v_{\text {lim }} / \sqrt{1-v_{\text {lim }}^{2}}\right)$. The limiting velocity is determined by the condition $\left(1-v_{\text {lim }}^{2}\right)^{1 / 4}=$ $T / T_{\text {diss }}+\left[\left(1-v_{0}^{2}\right)^{1 / 4}-1\right]$. The first term implements the relationship between $T$ and $v_{\text {lim }}$ that was heuristically motivated in Sec. 2. The second term accounts for the fact that the limiting velocity at $T_{\text {diss }}$ may be nonzero, as is the case in plasmas with a gravity dual. We choose $v_{0}=0.2$.

We also need the number of primordial $c \bar{c}$ pairs produced in the heavy-ion collision. This is proportional to the $c \bar{c}$ cross section in $p p$ collisions, which suffers from large uncertainties. For LHC energies, we use $d \sigma_{c \bar{c}}^{p p} / d y=$
$1850 \mu \mathrm{b}$, which is within the plausible range [26]. With this choice, one has $N_{c \bar{c}} \simeq 60$.

The results of numerically evaluating Eqs. (2) and (3) for LHC values of the parameters [27] and $T_{\text {diss }}=1.25 T_{c}$ and $M_{c}=1.7 \mathrm{GeV}$ are plotted in Fig. 5. Although the value of $M_{c}$ is relatively high, the values of $M_{c}$ and $T_{\text {diss }}$ are within the range commonly considered in the literature [28]. We have chosen them solely for illustrative purposes, since for these values the $J / \psi$ photons give rise to a clear peak. Varying these and other parameters in the model can decrease the sharpness of the peak and eventually turn it into a smooth enhancement distributed over a broad range of frequencies. An exhaustive and precise study of this effect for the many parameters in the model is beyond the scope of this Letter, but we note the following qualitative dependences: Decreasing the quark mass decreases the magnitude of the signal and turns the peak into an enhancement. Perhaps surprisingly, higher values of $T_{\text {diss }}$ also make the peak less sharp. Increasing the thermalization time decreases the background and hence increases the sharpness of the peak. Increasing the width $\Gamma$ by a factor of 2 turns the peak into an enhancement. And crucially, $S$ depends quadratically on the $c \bar{c}$ cross section. Since at RHIC energies this is believed to be 10 times smaller than at LHC energies, the enhancement discussed above is presumably unobservable at the RHIC.

The above considerations show that a precise study of the enhancement is not possible without a very detailed understanding of the in-medium dynamics of the $J / \psi$. On the other hand, they also illustrate that an order-one enhancement, or even a peak, in the spectrum of thermal photons produced by the QGP is possible. This thermal excess is concentrated roughly between 3 and $5 \mathrm{GeV}$. In this range the number of thermal photons at LHC is expected to be comparable or larger than that of perturbative QCD (pQCD) photons produced in initial partonic collisions [30]. Thus we expect the thermal excess to be observable even in the presence of the pQCD background.

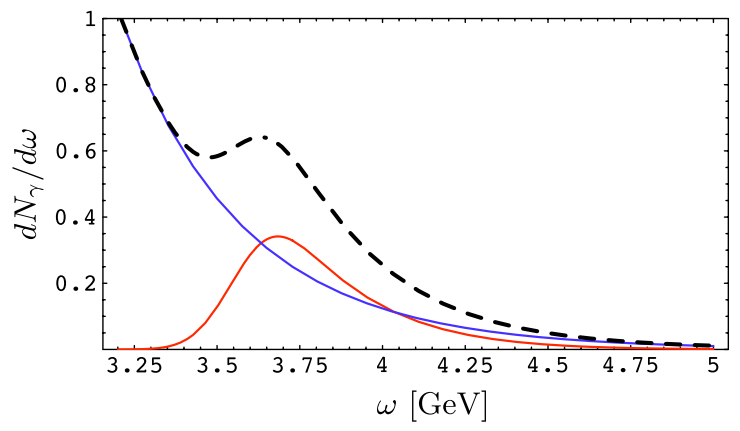

FIG. 5 (color online). Thermal photon spectrum. The (arbitrary) normalization is the same for all curves. The continuous, monotonically decreasing, blue curve is the background from light quarks. The continuous, red curve is the signal from $J / \psi$ mesons. The dashed, black curve is the sum of the two. 
We have examined the possibility of an analogous effect associated to the $Y$ meson. In this case, $\omega_{\text {peak }} \gtrsim 10 \mathrm{GeV}$. At these energies the number of thermal photons is exponentially smaller than that coming from $\mathrm{pQCD}$ photons, so an observable effect is less likely.

5. Conclusions. - Our two assumptions about the dynamics of vector mesons in the QGP can be motivated, and could in principle be checked, purely within QCD. We therefore emphasize that the prediction of a peak in the thermal photon spectrum does not rely on whether or not the QGP is well described by a dual gravity theory.

In practice, however, our ability to verify these assumptions in QCD is very limited due to the lack of well-suited tools. It is therefore useful to investigate if they hold for strongly coupled, large- $N_{c}$ plasmas with a gravity dual, for which the gravity description provides such a tool. In this Letter, we have demonstrated that both assumptions are indeed true for all such plasmas.

We thank M. Cacciari, D. d'Enterria, E. Ferreiro, S. Hartnoll, G. Martinez, V. Koch, M. Strassler, V. N. Tram, and L. Yaffe for discussions, the INT at the University of Washington for hospitality, and the DOE, Contract No. DE-AC03-76SF00098 (J.C.S.) and NSF Grant No. PHY-0244764 (D. M.), for support.

[1] T. Matsui and H. Satz, Phys. Lett. B 178, 416 (1986).

[2] O. Kaczmarek et al., Phys. Rev. D 70, 074505 (2004); 72, 059903(E) (2005); P. Petreczky and K. Petrov, Phys. Rev. D 70, 054503 (2004); O. Kaczmarek and F. Zantow, Phys. Rev. D 71, 114510 (2005); A. Mocsy and P. Petreczky, Phys. Rev. D 77, 014501 (2008); A. Mocsy, arXiv:0811.0337.

[3] M. Asakawa and T. Hatsuda, Phys. Rev. Lett. 92, 012001 (2004); S. Datta et al., Phys. Rev. D 69, 094507 (2004); T. Hatsuda, Int. J. Mod. Phys. A 21, 688 (2006); F. Karsch et al., Phys. Lett. B 637, 75 (2006); H. Satz, Nucl. Phys. A783, 249 (2007); M. Asakawa et al., Nucl. Phys. A715, 863 (2003); Nucl. Phys. B, Proc. Suppl. 119, 481 (2003).

[4] M. C. Chu and T. Matsui, Phys. Rev. D 39, 1892 (1989).

[5] K. Peeters et al., Phys. Rev. D 74, 106008 (2006); H. Liu et al., Phys. Rev. Lett. 98, 182301 (2007); M. Chernicoff et al., J. High Energy Phys. 09 (2006) 068; Q. J. Ejaz et al., J. High Energy Phys. 04 (2008) 089.

[6] An alternative possibility (not realized in plasmas with a gravity dual) would be that meson states with $k$ above some upper bound cease to exist. In any event, our conclusions will rely on meson states existing (with a sufficiently narrow width) only up to some moderate $k$ for which $\omega(k) \sim k$.

[7] D. Mateos et al., J. High Energy Phys. 05 (2007) 067.
[8] D. Mateos and L. Patino, J. High Energy Phys. 11 (2007) 025 .

[9] We assume that the meson is flavorless, and we neglect the thermal mass of the photon.

[10] In the vacuum, only the decay into a virtual photon would be allowed by kinematics. In the medium, the decay will take place unless the photon-meson coupling vanishes for some reason (e.g., a symmetry). We know of no such reason in QCD.

[11] E. Witten, Adv. Theor. Math. Phys. 2, 505 (1998).

[12] A. Karch and L. Randall, J. High Energy Phys. 06 (2001) 063; A. Karch and E. Katz, J. High Energy Phys. 06 (2002) 043.

[13] D. Mateos et al., Phys. Rev. Lett. 97, 091601 (2006).

[14] Specific examples of this transition in so-called $\mathrm{D} p / \mathrm{D} q$ systems were originally seen in Ref. [15]. More recently, similar transitions for $\mathrm{D} p / \mathrm{D} q / \mathrm{D} \bar{q}$ systems [16] have been found in Ref. [17].

[15] J. Babington, Phys. Rev. D 69, 066007 (2004); I. Kirsch, Fortschr. Phys. 52, 727 (2004); M. Kruczenski et al., J. High Energy Phys. 05 (2004) 041.

[16] T. Sakai and S. Sugimoto, Prog. Theor. Phys. 113, 843 (2005).

[17] O. Aharony et al., Ann. Phys. (N.Y.) 322, 1420 (2007); A. Parnachev and D. A. Sahakyan, Phys. Rev. Lett. 97, 111601 (2006).

[18] C. Hoyos-Badajoz et al., J. High Energy Phys. 04 (2007) 031.

[19] In the $\mathrm{D} p / \mathrm{D} q$ systems, this is because the bare quark mass is proportional to the distance between the $\mathrm{D} q$-branes and the $\mathrm{BH}$; in the $\mathrm{D} p / \mathrm{D} q / \mathrm{D} \bar{q}$ systems, this is because the chiral symmetry-breaking scale increases with the distance between the $\mathrm{D} q / \mathrm{D} \bar{q}$-branes and the $\mathrm{BH}$. In $\mathrm{D} p / \mathrm{D} q$ systems at finite baryon density, the branes always fall through the horizon, but for large quark masses the physics is essentially the same as at zero density [20].

[20] S. Kobayashi et al., J. High Energy Phys. 02 (2007) 016.

[21] R. Rapp and J. Wambach, Eur. Phys. J. A 6, 415 (1999).

[22] P. Braun-Munzinger and J. Stachel, Phys. Lett. B 490, 196 (2000); A. Andronic et al., Phys. Lett. B 571, 36 (2003).

[23] A. Andronic et al., Nucl. Phys. A789, 334 (2007).

[24] B. W. Zhang et al., Phys. Rev. C 77, 024901 (2008).

[25] Whose subsequent distribution is also thermal. This implies that the $J / \psi$ suppression is the same as in Ref. [23].

[26] M. Cacciari (private communication).

[27] $T(0)=470 \mathrm{MeV}, \quad z_{0}=0.3 \mathrm{fm}, \quad v_{z}=2 \times 0.7 c, \quad r_{0}=$ $6.6 \mathrm{fm}, a_{\perp}=0.085 c^{2} / \mathrm{fm}$, and $t_{\text {therm }}=0.21 \mathrm{fm} / c$.

[28] For the charm mass, a typical range is $1.3 \leq M_{c} \leq$ $1.7 \mathrm{GeV}$ because of a substantial thermal contributionsee, e.g., [29] and references therein. The value of $T_{\text {diss }}$ is far from settled, but a typical range is $T_{c} \leq T_{\text {diss }} \leq 2 T_{c}$ $[2,3]$.

[29] A. Mocsy and P. Petreczky, Phys. Rev. D 73, 074007 (2006).

[30] F. Arleo et al., arXiv:0707.2357. 